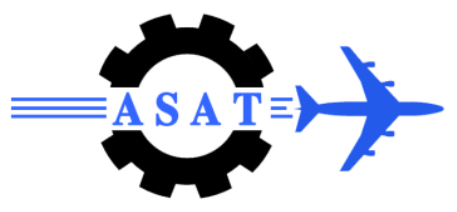

\title{
A Hybrid Algorithm for Speckle Noise Reduction in SAR Images
}

\author{
Nayef Y. Al-Delbah* and Kie B. Eom ${ }^{\dagger}$
}

\begin{abstract}
Synthetic Aperture Radar (SAR) images are usually impaired by Speckle Noise that appears as granules that result in degrading the fine details in the image. In order to reduce/remove such speckle noise, various processing algorithms have been considered in literature. In this paper, we introduce a Hybrid Algorithm based on spatial domain as well as frequency domain processing of SAR images so as to allow for better removal of speckle noise and preservation of the fine details. The proposed hybrid algorithm resulted in better than $30 \mathrm{~dB}$ improvement in the signal to noise ratio; i.e. in significant reduction/removal of most of the speckle noise from the image while preserving of image's fine details. The performance of the implemented hybrid algorithm for speckle noise reduction has been compared with the published respective performance results obtained by other algorithms. Pros and cons of various algorithms are also given along with conclusions and directions for future work.
\end{abstract}

Keywords: Synthetic Aperture Radar (SAR), speckle noise in SAR images, image processing, nonlinear diffusion, anisotropic diffusion, nonlinear filtering, discrete wavelet transform, adaptive filtering, and hybrid algorithm.

\section{Introduction}

Synthetic Aperture Radar (SAR) is an active coherent imaging technology, recording both the amplitude and the phase of the back-scattered radiation. The phases of the return signals from scatterers are randomly distributed and by the resulting random interference between the coherent returns, a sort of noise speckle is generated; thus degrading the image fine details giving the image the grainy appearance. Hence, SAR images suffer a grainy texture pattern that complicates image analysis and interpretation in remote sensing applications [1]. Speckle filtering is not only a common trend in most SAR image applications, but turns to be a critical step before edge detecting and object recognition for the intended targets [2].

Literature includes a wealth of different algorithms for speckle noise removal. A variety of algorithms has been proposed to restore noisy SAR images based on spatial domain filtering and adopted the hypothesis that speckle noise is essentially a multiplicative noise. Classical filtering techniques, such as the Wiener filter [3], proved to be insufficient as such filter is designed on the basis of assumption of independent additive noise while speckle noise is signal-dependent. Among the most well-known models are algorithms proposed by Lee [4],

\footnotetext{
"Ph.D. Candidate at the Electrical and Computer Engineering Department, School of Engineering and Applied Science of the George Washington University, Washington, DC 80305 USA. (naif@gwmail.gwu.edu).

$\dagger$ Professor at the Electrical and Computer Engineering Department, School of Engineering and Applied Science of the George Washington University, Washington, DC 80305 USA.
} 
Kuan et al. [5] and Frost et al. [6]. The adaptive method used by Kuan et al. and Frost et al. is to use a suitable neighborhood of the pixel for calculating statistical measures (e.g. mean and variance) to update the central pixel according to a filter based on those statistics. Another filtering technique adopted a "product" model as the basis for the development of the filter,such as the maximum a posteriori (MAP) Bayesian estimation proposed by Kuan et al. [7], the Gamma filter of Touzi et al. [8], and the model-based despeckling filter of Walessa et al. [9]. Foucher et al. [10] combines image multiscale analysis (wavelets) and classical techniques of adaptive filtering such as the Gamma MAP filter proposed by Lopes et al. [11]. In this approach the wavelet coefficients of the ground backscatter are estimated with a Bayesian model, maximizing the a posteriori probability density function and the different probability density function are modeled with the Pearson family of distributions.

Linear filtering techniques may result in some problems, such as blurring the sharp edges, destroying lines and other finer image details. They generally fail to effectively remove heavy noise. Due to these facts, nonlinear filtering such as wavelet transform is necessary. But, in wavelet thresholding the problem experienced is generally smoothening of edges.

In the present work, a Hybrid filtering Algorithm is proposed to invoke both spatial and frequency domains analysis of the speckled image. Frequency domain processing is first applied to the image by utilizing the discrete wavelet transform (DWT) and then the result is subsequently processed by an effective spatial domain filter. The performances are evaluated in terms of peak signal to noise ratio (PSNR) and mean square error (MSE). The correlation coefficient between the speckled image and the despeckled image is also computed.

The paper is organized as 5 sections including conclusions and direction for future work.

Section 2 describes the proposed hybridization approach while more detailed presentation of both frequency domain and spatial domain processing is given in section 3. Section 4 is devoted to the hybrid filtering implementation and assessment including a comparative evaluation of a variety of hybridization combinations. Finally, conclusions and future work are given in section 5 .

\section{Hybrid Approach for Speckle Denoising}

Speckle filtering models incorporate certain assumptions about speckle, scene, and observed signals; which influence scene reconstruction performed using an inversion of these models. Reconstruction methods based on the Kuan et al. [5] multiplicative speckle model exhibit better results than the ones based on the more restrictive, Lee [4] models. Multiplicative model assumes that the observed degraded/speckled image, $S$, and the noise-free original image, $I$, are related as follows:

$S(n, m)=I(n, m) u(n, m)$

where $u$ represents the multiplicative speckle noise with both mean and standard deviation of unit value.

This suggests denoising the image on the basis of homomorphism approach that takes the advantage of logarithmic transformation that is applied to Eq. (1) that converts the multiplicative form of noise into additive form. However, experiments showed that homomorphism affords no clear advantage compared to other methods that, in general, perform speckle filtering through application of a sliding window of fixed size to the image.

A different approach is proposed in the present work; that is the Hybrid approach depicted in the following figure Fig.1: 


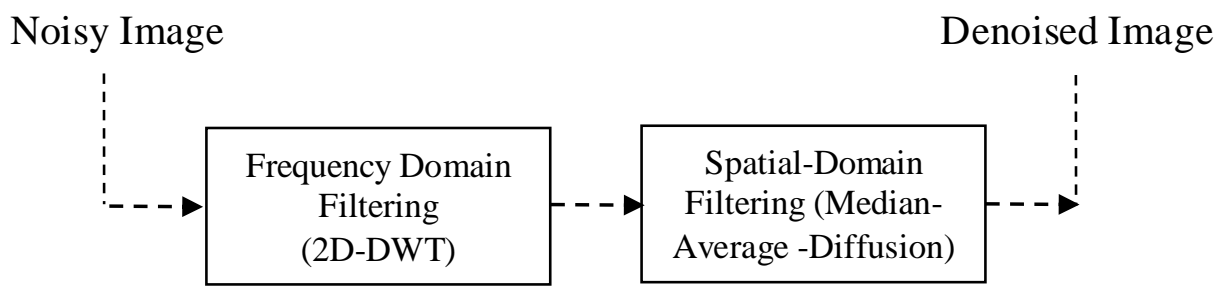

Fig. 1 Hybrid Denoising Configuration

Frequency domain analysis of SAR images is done first through Wavelet transform (WT) for denoising while preserving signal characteristics. Two-dimensional (2D) discrete wavelet transform (DWT) can be computed by filtering (low-pass and high-pass) the image in all directions and sub-sampling after applying the filters.

The advantage of WT is its representation for the image in a non-redundant form [12] but on the other hand, shift dependency is a disadvantage of WT; as a complete change in the transform coefficients occurs by a simple shift in the input image resulting in fused images that are non-consistent. Overcoming shift dependency can be accomplished by decomposing the input image so that it is represented as a shift invariant; this can be achieved by computing WT for all circular shifts. Another simpler way is applying the decomposition process on the sub-sampling (by dropping) and at each level of the decomposition; filters are modified, this will result in an efficient representation of a redundant signal [13].

The output of the frequency domain analysis will be subsequently analyzed using spatial domain analysis filters. Three types of filters were chosen to utilize their efficiency in the removal of speckle noise. They are diffusion, median and average filters in a cascaded form with the aim to improve the denoising result.

\section{Frequency Domain and Spatial Domain Processing}

In the following, presentation of both frequency domain and spatial domain denoising approaches are given.

\subsection{Frequency Domain Analysis}

For, wavelet function is used which is a form of mathematical functions that is defined over a finite interval and having zero average; and wavelets are used to transform arbitrary functions into several frequency components. Such type of functions is represented as a superposition of the wavelets set. The wavelet decomposition process involves three basic steps as: i) a linear forward wavelet transform; ii) nonlinear thresholding step and; iii) a linear inverse wavelet transform.

\subsubsection{Image Representation}

Let $S=\left\{S_{i j}, i, j=1,2, \ldots, M\right\}$ denote the $M \times M$ matrix of the speckled image and $M$ is an integer power of 2. Our goal is to estimate the signal $I$ from noisy observations $S$ such that Mean Squared Error (MSE) is minimum and Peak Signal to Noise Ratio (PSNR) is maximum. Let $W$ and $W^{-1}$ denote the two dimensional orthogonal discrete wavelet transform (DWT) matrix and its inverse respectively. Then $Y=W \cdot S$ represents the matrix of wavelet coefficients of $S$ having four divisions $(L L, L H, H L$, and $H H)$. Let $J$ be the total number of decompositions and $\mathrm{k}=1,2, \ldots, \mathrm{J}$ be the divisions' scale, and then the divisions $\mathrm{HH}_{\mathrm{k}}, \mathrm{HL}_{\mathrm{k}}$, and $\mathrm{LH}_{\mathrm{k}}$ are called details. The size of the divisions' scale, $k$, is $N / 2^{k} \times N / 2^{k}$. The division $L L_{J}$ is the low resolution residue. The wavelet denoising method processes each coefficient of $Y$ from the detail divisions with a threshold function to obtain $\widehat{\mathrm{X}}$. The denoised estimate is the inverse transform, $\hat{I}=W^{-1} \cdot \hat{X}[14]$. 


\subsubsection{Thresholding Techniques}

In various signals, energy is concentrated in a small number of dimensions and the coefficients of these dimensions are relatively large compared to any other signal, especially noise, which has its energy spread over a large number of coefficients. Hence, in wavelet, each coefficient is set to zero (threshold) by comparing against a threshold to eliminate noise, while preserving important information of the original signal [15]. There are two types of thresholding techniques: hard and soft thresholding. Hard thresholding is a keep-or-kill procedure and its operator is defined as:

$\varphi(t, \lambda)= \begin{cases}t & ,|t|>\lambda \\ 0 & , \text { otherwise }\end{cases}$

Soft thresholding shrinks the magnitudes of the coefficients above the threshold in absolute value and its operator is defined as:

$\varphi(t, \lambda)=\left\{\begin{array}{cc}0 & ,|t| \leq \lambda \\ \operatorname{sgn}(t)(|t|-\lambda) & , \text { otherwise }\end{array}\right.$

In our work, we are using Haar's mother wavelet, which can be described as:

$w(t)=\left\{\begin{array}{cc}1 & 0 \leq t<1 / 2 \\ -1 & 1 / 2 \leq t<1 \\ 0 & \text { otherwise }\end{array}\right.$

and its scaling function, $\varphi(t)$, can be described as:

$\varphi(t)=\left\{\begin{array}{cc}1 & 0 \leq t<1 \\ 0 & \text { otherwise }\end{array}\right.$

\subsubsection{Disadvantages}

The thresholding techniques have some underlying disadvantages. For instance, the estimated wavelet coefficients by the hard thresholding method are not continuous which may lead to the oscillation of the reconstructed signal. In the soft thresholding case, there are deviations between image coefficients and thresholded coefficients which influence the accuracy of the reconstructed signal. Retention of the edges is also a problem here. Different edge detection algorithms are used to extract the contour feature of the image. Determination of the threshold's value is critical as larger value may result into loss of information while smaller one may allow noise to continue.

\subsection{Spatial Domain Analysis}

The output of the frequency domain analysis will be subsequently analyzed using a combination of the following spatial domain analysis filters.

\subsubsection{Diffusion Filter}

Keeping the balance between speckle suppression and feature preservation is a key point in effective speckle noise removing. Diffusion (or Anisotropic Diffusion) filter is a powerful filter where local image variation is measured at every point, and pixel values are averaged from neighborhoods whose size and shape depend on local variation. Such filters have been recently used for the speckle reduction and removal in the ultrasound images; this filter has excellent speckle noise reduction and edges preserving due to its nonlinearity nature and the adaptive anisotropy $[16,17]$. It is to be noted that Diffusion methods average over extended regions by solving partial differential equations similar to heat diffusion equation. 


\subsubsection{Median Filter}

Median filter is a windowed filter of nonlinear class that removes noise while preserving edges. This type of filters checks all the pixels in the image and replaces with the median neighboring pixels. By sorting the pixel values in the image into numerical order and the middle pixel value is the median value and used for replacing the considered pixel. This filter is good in speckle noise removal and edge preserving for the image enhancement [18]. The advantage of the median filter is its simplicity and algorithmic straightforwardness. But due to its nonadaptive nature it deteriorates not only speckles but details as well. Diffusion filter is more effective technique though more complicated

\subsubsection{Average (Mean) Filter}

Average filter is a windowed filter of linear classes that smoothes image and works as lowpass filter. The average value is calculated by finding out the sum of all pixels in a window and then dividing the sum by the number of pixels, after that spatial filtering is performed on each pixel in the center of the window [19]. Average filter is used to filter out the noise involved and to enhance the image visualization and interpretation.

\section{Hybrid Denoising Implementation and Assessment}

The algorithms presented in section 3 have been implemented and their performance has been evaluated on the basis of

- the mean square error (MSE) as well as the peak signal to noise ratio (PSNR).

- Correlation between the speckled image, $S$, and the despeckled image, $I$.

\subsection{Mean Square Error and Signal to Noise Ratio}

The mean square error (MSE) gives the average square difference of pixels between the speckled image, $S$, and the despeckled image, $I$. The lower the value of MSE is, the lower the error is.

$M S E=\frac{1}{M N} \sum_{i=1}^{M} \sum_{j=1}^{N}[I(i, j)-S(i, j)]^{2}$

where $M$ and $N$ are the image size. The peak signal to noise ratio (PSNR), in decibels, is commonly used to measure the quality of an image denoising. The greater the PSNR is, the better the speckle reduction algorithm is. However, this statement has a limitation. We prefer not to have PSNR that is greater than $35 \mathrm{~dB}$ as this tends to blur the image and destroys the fine details. The PSNR can be described as:

$P S N R=10 \log _{10}\left(\frac{P_{\text {max }}{ }^{2}}{M S E}\right)$

where $P_{\max }$ is the maximum possible value of the input pixel.

The highest PSNR of a cascading may refer to the best noise removal. However, choosing a cascade of a value higher than $35 \mathrm{~dB}$ is not preferable because some of the image contents will not be seen clearly. In addition, some of the image details will be removed [20].

\subsection{Correlation Coefficient}

The third assessment method is to compute the 2D correlation coefficient, $\rho$, between the speckled image, $S$, and the despeckled image, $I$. 


$$
\rho=\frac{\sum_{i=1}^{M} \sum_{j=1}^{N}\{[I(i, j)-\bar{I}][S(i, j)-\bar{S}]\}}{\sqrt{\left\{\sum_{i=1}^{M} \sum_{j=1}^{N}[I(i, j)-\bar{I}]^{2}\right\}\left\{\sum_{i=1}^{M} \sum_{j=1}^{N}[S(i, j)-\bar{S}]^{2}\right\}}}
$$

where $\bar{I}$ and $\bar{S}$ are the mean values of $I$ and $S$, respectively. If the value of the correlation coefficient is +1 , then it indicates a perfect positive linear relationship, and if it is -1 , then it indicates a perfect decreasing linear relationship. For a value between -1 and 1 , it indicates the degree of linear dependency. As correlation coefficient approaches zero there is less of a relationship. The closer the coefficient is to either -1 or +1 , the stronger the correlation between the variables.

\subsection{Discussions and Comparative Evaluation of Hybridization Schemes}

Fig. 2 shows a raw SAR image and the output of the three decomposition levels of 2D-DWT that's applied on the raw SAR image, and then 2D-IDWT is applied. The speckle noise accumulating in the high frequencies of the image are filtered out. The output image is assessed against the speckled image using correlation coefficient, MSE, and PSNR. The results are $0.8690,405.60$, and $22.05 \mathrm{~dB}$ respectively.
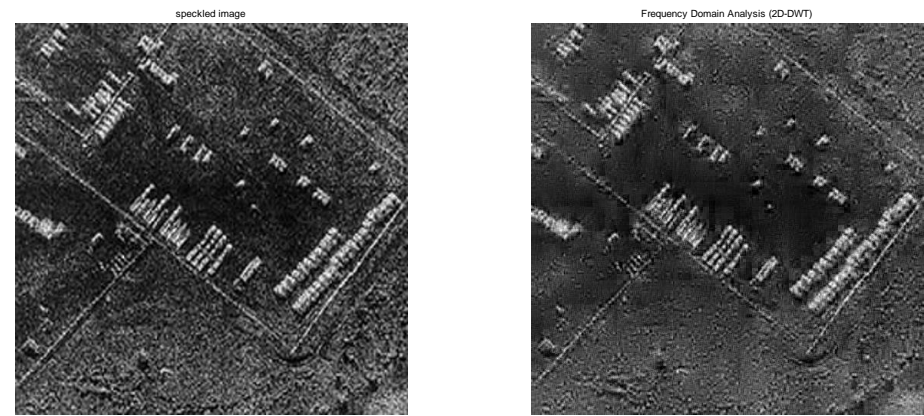

\section{Fig. 2 (Left) Raw SAR Image, (right) Result of Frequency Domain Analysis}

In the spatial domain analysis, first, the chosen filters are directly and separately applied to the same raw SAR image to show how effective these filters are. Table 1 shows correlation coefficient, MSE, and PSNR respectively. Elapsed time is 0.72 seconds.

Table 1 Results Values for Spatial Domain Analysis

\begin{tabular}{c|c|c|c|c}
\hline \hline Spatial Domain Filter & Correlation Coefficient & MSE & PSNR & Time (s) \\
\hline Average & 0.9835 & 67.08 & 29.87 & 0.16 \\
\hline Median & 0.9848 & 56.26 & 30.63 & 0.23 \\
\hline Diffusion & 0.9876 & 55.77 & 30.67 & 1.28 \\
\hline
\end{tabular}
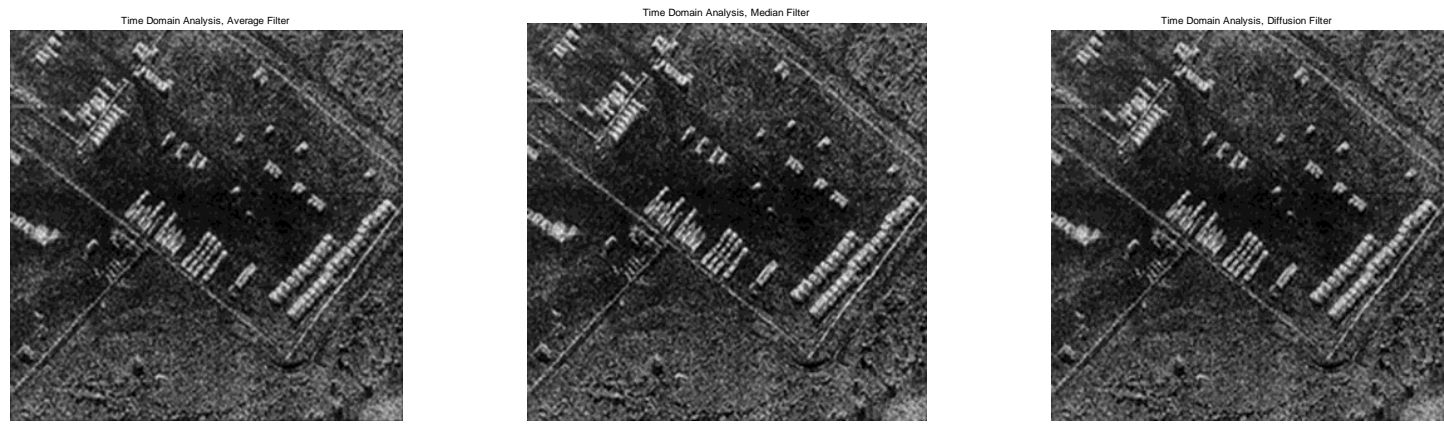

Fig. 2 (Left to right) Frequency Domain Analysis: Average, Median, and Diffusion 
The result from the frequency domain analysis is further filtered in the spatial domain with a cascaded filter for better speckle removal. Fifteen different spatial domain filter models were examined. The choice of a particular model is based on the correlation coefficient as well as the PSNR values. Four models are rejected as they did not yield the desired improvement, although, one can note that the MSE value has been enhanced in the last two models. Table 2 shows values obtained from these four models.

Table 2 Results Values for Rejected Cascading

\begin{tabular}{l|c|c|c|c}
\hline \multicolumn{1}{c|}{ Model } & Correlation Coefficient & MSE & PSNR & Time (s) \\
\hline \hline Wavelet $\rightarrow$ Average & 0.9473 & 137.24 & 27.68 & 0.82 \\
\hline Wavelet $\rightarrow$ Median & 0.9486 & 131.86 & 27.86 & 0.95 \\
\hline Wavelet $\rightarrow$ Diffusion $\rightarrow$ Average & 0.9661 & 69.86 & 29.69 & 1.35 \\
\hline Wavelet $\rightarrow$ Diffusion $\rightarrow$ Median & 0.9693 & 62.68 & 30.16 & 1.71 \\
\hline \hline
\end{tabular}

Although, the correlation coefficient and MSE are highly improved, we rejected five other models based on the $35 \mathrm{~dB}$ threshold. Table 3 shows the output of these filters.

Table 3: Results Values for Rejected Cascading Based on the 35dB Threshold

\begin{tabular}{l|c|c|c|c}
\hline \multicolumn{1}{c|}{ Model } & Correlation Coefficient & MSE & PSNR & Time (s) \\
\hline \hline Wavelet $\rightarrow$ Average $\rightarrow$ Diffusion $\rightarrow$ Median & 0.9936 & 13.78 & 36.10 & 1.78 \\
\hline Wavelet $\rightarrow$ Average $\rightarrow$ Median & 0.9930 & 15.20 & 36.30 & 0.94 \\
\hline Wavelet $\rightarrow$ Median $\rightarrow$ Average $\rightarrow$ Diffusion & 0.9941 & 12.54 & 36.82 & 1.80 \\
\hline Wavelet $\rightarrow$ Diffusion $\rightarrow$ Average $\rightarrow$ Median & 0.9959 & 7.57 & 38.63 & 1.81 \\
\hline Wavelet $\rightarrow$ Average $\rightarrow$ Median $\rightarrow$ Diffusion & 0.9964 & 6.57 & 39.21 & 1.47 \\
\hline
\end{tabular}

Table 4 shows the output of the remaining six different models. In general, one may note that when using average filter at the end of any model, it does not add a remarkable improvement. Thus, it is recommended to drop out such model so that the computation complexity is reduced. This leaves us always with models that end with diffusion filter. It can be seen that the last cascade is the best for speckle removal from SAR images. Hence, a proper spatial domain filtering technique would be (Average $\rightarrow$ Diffusion). By combining this spatial domain model with the wavelet decomposition process in the frequency domain analysis, hence we introduce the hybrid algorithm for speckle noise reduction in SAR Images (Wavelet $\rightarrow$ Average $\rightarrow$ Diffusion). The correlation value obtained at the end of the hybrid algorithm is 0.9916 and the final PSNR obtained is $35 \mathrm{~dB}$, which suggest that the obtained results are accurate and approaching perfectness.

Table 4 Results Values for Recommended Cascading

\begin{tabular}{l|c|c|c|c}
\hline \multicolumn{1}{c|}{ Model } & Correlation Coefficient & MSE & PSNR & Time (s) \\
\hline \hline Wavelet $\rightarrow$ Diffusion & 0.9848 & 49.56 & 32.11 & 1.51 \\
\hline Wavelet $\rightarrow$ Median $\rightarrow$ Average & 0.9834 & 36.40 & 32.69 & 0.87 \\
\hline Wavelet $\rightarrow$ Diffusion $\rightarrow$ Median $\rightarrow$ Average & 0.9886 & 21.39 & 34.34 & 1.69 \\
\hline Wavelet $\rightarrow$ Median $\rightarrow$ Diffusion & 0.9904 & 23.93 & 34.51 & 1.63 \\
\hline Wavelet $\rightarrow$ Median $\rightarrow$ Diffusion $\rightarrow$ Average & 0.9899 & 18.61 & 34.76 & 1.75 \\
\hline Wavelet $\rightarrow$ Average $\rightarrow$ Diffusion & 0.9916 & 20.28 & 35.04 & 1.56 \\
\hline \hline
\end{tabular}

Figure 3 shows the speckled raw SAR image used, and the output of the hybrid algorithm at each stage: three decomposition levels of 2D-DWT and 2D-IDWT where the speckle noise accumulating in the high frequencies of the image are filtered out, then the output image of the spatial domain filters, average and diffusion respectively. 

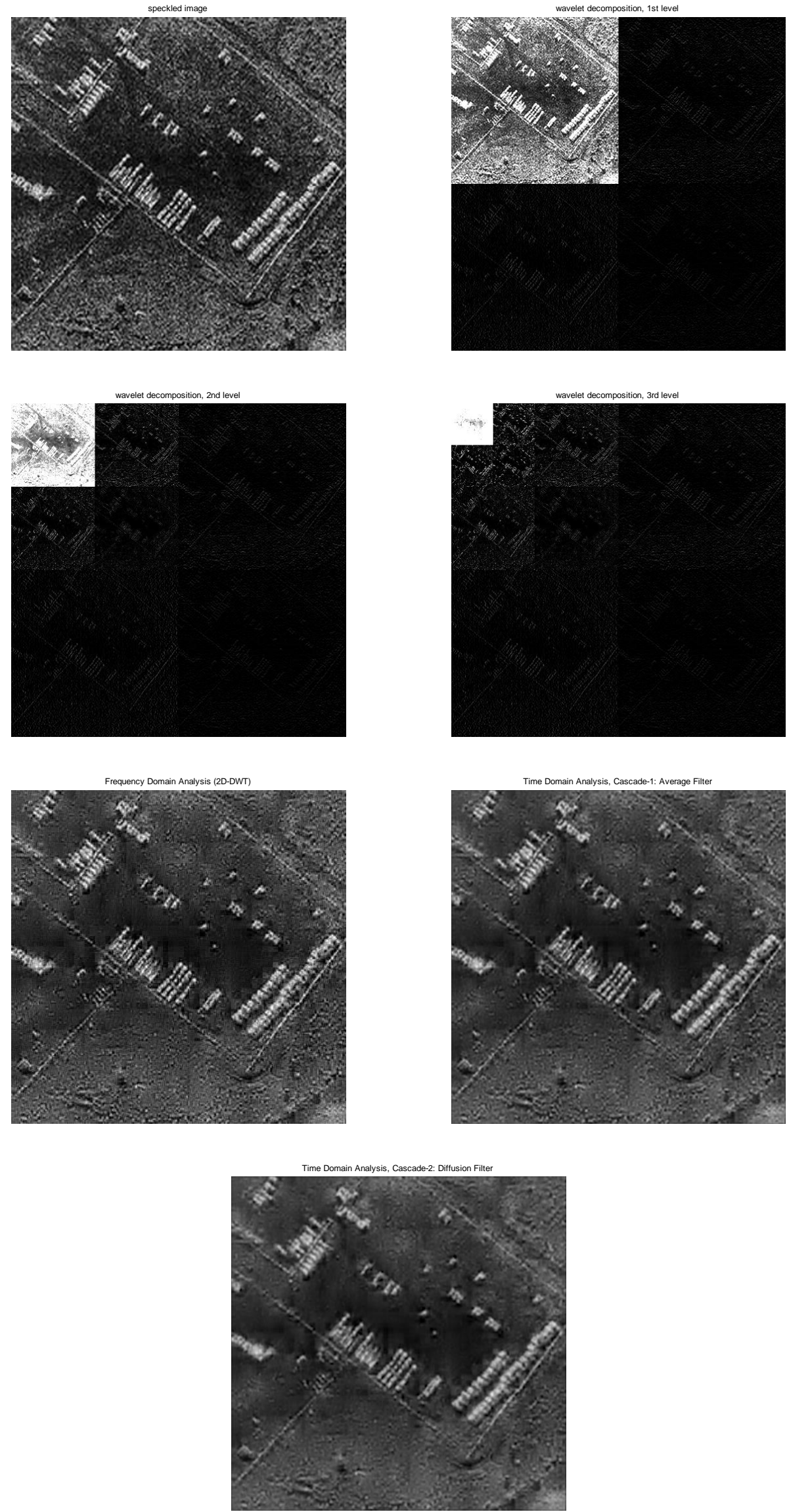

Fig. 3 The Output of the Hybrid Algorithm at Each Stage:

Wavelet, Average, and Diffusion 


\section{Conclusions and Future Work}

In this paper, a hybrid algorithm is proposed for removal of speckle noise that degrades the fine details of the SAR images. The hybrid algorithm consists of two stages; frequency domain and spatial domain analyses. Frequency domain analysis uses discrete wavelet transform to remove high frequencies in speckle noise. Spatial domain analysis uses a cascaded filter (involving two filters: average and diffusion) for improving removal of the speckle. Evaluation of the accuracy of the implemented method was done on the bases of peak signal-to-noise ratio and the input-output cross-correlation coefficient. Results prove the powerful performance and effectiveness of the hybrid algorithm as it yields a value for the PSNR that ranges between $30 \mathrm{~dB}$ and $35 \mathrm{~dB}$.

In order to show the effectiveness of the proposed algorithm, several other statistical filters, homomorphism-based statistical filters, and other commonly used thresholding techniques are to be contrasted: performance-wise and complexity-wise. The expected drawback of the proposed algorithm is its computational complexity, hence processing time. With the advancement in computational technology, a slower execution problem would be solved by concurrent processing, fast algorithms and parallelism. This is a line in the future work directions after comparative analysis of viable processing algorithms.

\section{References}

[1] U. Soergel, S. Schulz, U. Thoennessen, and U. Stilla, "Integration of 3D Data in SAR Mission Planning and Image Interpretation in Urban Areas," Information Fusion, Vol. 6, No. 4, Dec. 2005, pp. 301-310.

[2] J.-S. Lee, K. Hoppel and S.A. Mango, "Unsupervised Estimation of Speckle Noise in Radar Images," International Journal of Imaging Systems and Technology, Vol.4, No. 4, 1992, pp. 298-305.

[3] N. Wiener, "Extrapolation, Interpolation, and Smoothing of Stationary Time Series," Cambridge, MA: The MIT Press, 1964.

[4] J.-S. Lee, "Digital Image Enhancement and Noise Filtering by Use of Local Statistics," IEEE Transactions on Pattern Analysis and Machine Intelligence, Vol. 2, No. 2, Mar. 1980, pp. 165-168.

[5] D. T. Kuan, A. A. Sawchuk, T. C. Strand, and P. Chavel. "Adaptive Noise Smoothing Filter for Images with Signal-Dependent Noise," IEEE Transactions on Pattern Analysis and Machine Intelligence, Vol. 7, No. 2, Mar. 1985, pp. 165-177.

[6] V. S. Frost, J. A. Stiles, K. S. Shanmugan, and J. C. Holtzman, "A Model for Radar Images and its Application to Adaptive Digital Filtering of Multiplicative Noise," IEEE Transactions on Pattern Analysis and Machine Intelligence, Vol. 4, No. 2, Mar. 1982, pp. 157-166.

[7] D. T. Kuan, A. A. Sawchuk, T. C. Strand, and P. Chavel, "Adaptive Restoration of Images with Speckle," IEEE Transactions on Acoustics, Speech, and Signal Processing, Vol. 35, No. 3, Mar. 1987, pp. 373-383.

[8] R. Touzi, S. Coze, T. Le Toan, and A. Lopes, "Optimization of Polarimetric Discriminators In SAR Images," Geoscience and Remote Sensing Symposium, District of Columbia, USA, May. 1990, pp. 1683-1686.

[9] M. Walessa and M. Datcu, "Model-Based Despeckling and Information Extraction from SAR Images," IEEE Transactions on Geoscience and Remote Sensing, Vol. 38, No. 5, Sep.2000, pp. 2258-2269.

[10] S. Foucher, G.B. Bénié, J-M. Boucher, "Multiscale MAP Filtering of SAR Images", IEEE Transactions on Image Processing, Vol. 10, No. 1, Jan. 2001, pp.49-60.

[11] A. Lopes, E. Nezry, R. Touzi and H. Laur, "Structure detection and statistical adaptive speckle in SAR images", International Journal of Remote Sensing, 1993, pp.1735-1758. 
[12] L. Gagnon and A. Jouan, "Speckle Filtering of SAR Images: A Comparative Study Between Complex-Wavelet Based and Standard Filters," SPIE Proceedings of Wavelet Applications in Signal and Image Processing V, California, USA, Vol. 3169, 30 Jul. 1997, pp. 80-91.

[13] N. Agrawal and K. Venugopalan, "SAR Image Compression Using Wavelet Packets," IEEE Proceedings of International Conference on Signal Processing Systems, Singapore, 15-17 May 2009, pp. 343-347.

[14] J. Maycock, B.M. Hennelly, J.B. McDonald, Y. Frauel, A. Castro, B. Javidi, and T.J. Naughton, "Speckle Reduction Using The Discrete Fourier Filtering Technique," IEEE Proceedings of International Conference on Machine Vision and Image Processing, Kildare, Ireland, 05-07 Sep. 2007, pp. 201-201.

[15] L. Kun, W. Shuang, H. Biao, and J. Licheng, "An Algorithm for SAR Image Embedded Compression Based On Wavelet Transform," IEEE Proceedings of International Conference on Software Engineering, Artificial Intelligence, Networking, and Parallel/Distributed Computing, Qingdao, China, 30 Jul. - 01 Aug. 2007, pp. 374-378.

[16] K. Krissian, C.-F. Westin, R. Kikinis and K. Vosburgh, "Oriented Speckle Reducing Anisotropic Diffusion," IEEE Transactions on Image Processing, Vol. 16, No. 5, May 2007, pp. 1412-1424.

[17] Xiaona Zhi, Tianfu Wang; "An Anisotropic Diffusion Filter For Ultrasonic Speckle Reduction," IEEE Proceedings of International Conference on Visual Information Engineering, Xian China, 29 Jul. -01 Aug. 2008, pp. 327-330.

[18] W. Hagg and M. Sties, "Efficient Speckle Filtering of SAR Images," IEEE Proceedings of International Geoscience and Remote Sensing Symposium, 08-12 Aug. 1994, pp. 2140-2142.

[19] Zhao Chunhong, Liu Mingyu, Ji Hongbing, "A New Speckle Reduction Method of Medical Ultrasonic Image," IEEE Proceedings of International Conference on Bioinformatics and Biomedical Engineering, Wuhan, China, 06-08 Jul 2007, pp. 10041006.

[20] J.M. Park, W.J. Song, W.A. Pearlman, "Speckle Filtering of SAR Images Based on Adaptive Windowing," IEEE Proceedings of Vision, Image and Signal Processing, Vol. 146, No. 4, Aug. 1999, pp. 191-197. 